RASĀYAN J. Chem.

Vol. 13 | No. 1 |676 - 683| January - March | 2020 ISSN: 0974-1496 | e-ISSN: 0976-0083 | CODEN: RJCABP

RJC http://www.rasayanjournal.com http://www.rasayanjournal.co.in

\title{
SYNTHESIS OF NOVEL PYRAZOLINES AND THEIR ANTIMICROBIAL ACTIVITY
}

\author{
S. Sathiya ${ }^{1}$, A. Keerthika ${ }^{1}$ B. S. Krishnamoorthy ${ }^{1}$, S. Nandhabala ${ }^{1}$, \\ S. Aravind ${ }^{2}$, N. Hari ${ }^{3}$ and R. Ravikumar ${ }^{1, *}$

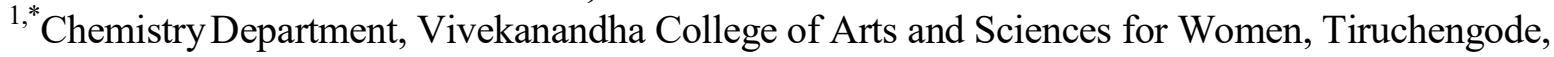 \\ 637205, Tamilnadu, India \\ ${ }^{2}$ School of Chemical and Biotechnology, SASTRA Deemed University, Thirumalaisamudram, \\ Thanjavur, 613401, Tamilnadu, India \\ ${ }^{3}$ NMR Laboratory, School of Chemical and Biotechnology, SASTRA Deemed University, \\ Thirumalaisamudram, Thanjavur, 61340, Tamilnadu, India,. \\ *E-mail: drravikumarvicas@gmail.com
}

\begin{abstract}
A series of novel 4-nitroaniline derived pyrazolines have been synthesized and characterized by data arrived from the analytical methods such as FT-IR, ${ }^{1} \mathrm{H}$ NMR, ${ }^{13} \mathrm{C}$ NMR and Mass spectral analysis. X-ray crystallographic structure elucidation of a crystalline compound 4-fluoro benzaldehyde derived chalcone is presented. The impact of electronegativity and extension of conjugation offered by the nitro group in the synthesized molecule on its biological activity was analyzed. Title compounds were subjected to antimicrobial activity.

Keywords: Chalcones, Pyrazolines, XRD, Antimicrobial activity. 4-nitro aniline derived triazoles, electronegativity, extend of conjugation.

(C) RASĀYAN. All rights reserved
\end{abstract}

\section{INTRODUCTION}

The diverse pharmacological activity of pyrazoline linked heterocyclic compounds was well established. A modified pyrazoline ring linked to other heterocyclic moieties has shown potent biological activity with lesser toxicity. The 2-pyrazolines constituted heterocyclic linked derivatives possess anticancer ${ }^{1}$, antidepressant and anticonvulsant ${ }^{2}$, Cannabinoid receptor antagonist activity ${ }^{3}, \mathrm{COX}-2$ inhibitor activity ${ }^{4}$, antihepatotoxic activity $^{5}$, antihyperglycemic activity $^{6}$, anti-inflammatory and analgesic activity ${ }^{7,8,9}$, antimalarial activity ${ }^{10}$, antioxidation property ${ }^{11}$, MAO inhibitors ${ }^{12}{ }^{13}$, antitubercular activity ${ }^{14,15}$, antimicrobial activity. ${ }^{16,}{ }^{17}$ From literature review it was found that pyrazolines linked to different chemical moieties viz., benzofuran, benzene sulphonamide, pyrimidine, coumarine, carboxamide and benzo pyrrole, 1, 2, 4-triazol, 1, 4-dioxane, quinozoline, benzene sulphonyl urea and thiourea, carbohydrazide and thiazole have been studied to some extent with their biological activity. Hitherto only a very few compounds of this class like 4-methyl-5-(5-phenyl-4, 5-dihydro-1H-pyrazole-3-yl)-1-(p-tolyl)$1 \mathrm{H}-1,2,3$-triazole have been reported in the literature. ${ }^{16} \mathrm{We}$, herein, report the synthesis and antimicrobial activity of a series of 1,2,3-triazole linked chalcone and their pyrazolines following Scheme-1. Out of ten chalcones prepared, 4-F benzaldehyde derived chalcone was induced to crystallize and its XRD pattern is presented.

\section{EXPERIMENTAL}

Solvents available commercially were used after purification by the distillation process. Reaction completion was monitored by both silica gel coated glass plates and pre-coated Aluminium TLC plates procured from Merck (Germany). Purified compounds were characterized by elemental analysis $\mathrm{IR},{ }^{1} \mathrm{H}$ NMR, ${ }^{13} \mathrm{C}$ NMR and an EI-mass spectrometry. IR spectra were recorded in $\mathrm{KBr}$ on a Perkin-Elmer model

Rasayan J. Chem., 13(1), 676-683(2020)

http://dx.doi.org/10.31788/RJC.2020.1315568

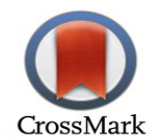


RASĀYAN J. Chem.

Vol. 13 | No. 1 |676 - 683| January - March | 2020

1620 FTIR spectrophotometer, ${ }^{1} \mathrm{H}$ NMR and ${ }^{13} \mathrm{C}$ NMR spectra were recorded in Brucker AvanceII 300 $\mathrm{MHz}$ spectrophotometer using $\mathrm{CDCl}_{3} / \mathrm{DMSO}$ as a solvent and mass spectra were recorded on a JEOL mass spectrometer. XRD was measured using CrysAlisPro (CrysAlisPro 1.171.39.46 (Rigaku Oxford Diffraction, 2018).

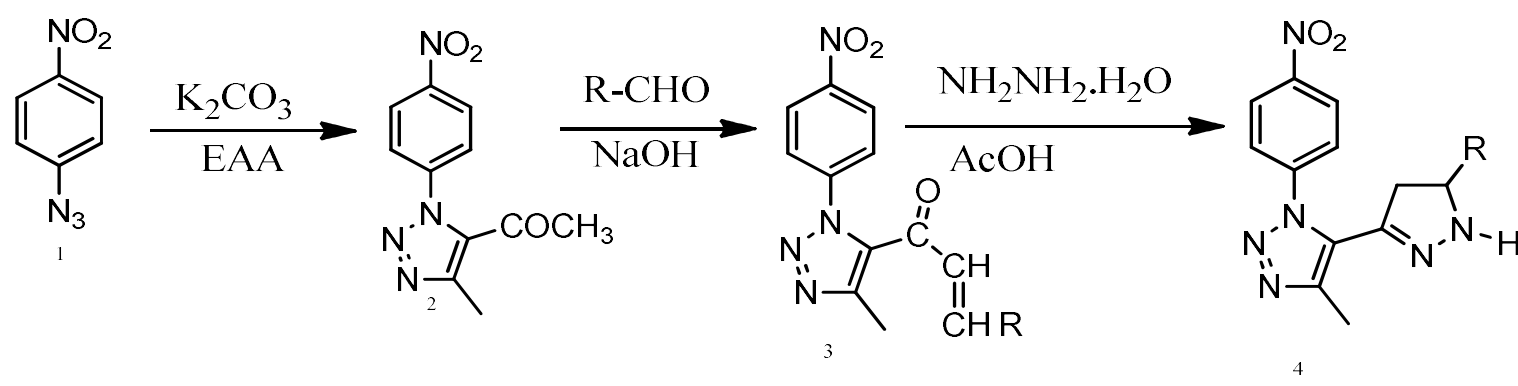
R: In 3 and 4
1. $\mathrm{C}_{6} \mathrm{H}_{5}$
7. $3-\mathrm{ClC}_{6} \mathrm{H}_{4}$
$2.4-\mathrm{OCH}_{3} \mathrm{C}_{6} \mathrm{H}_{4}$
8. $4-\mathrm{ClC}_{6} \mathrm{H}_{4}$
3. $4-\mathrm{NO}_{2} \mathrm{C}_{6} \mathrm{H}_{4}$
9. $2-\mathrm{OHC}_{6} \mathrm{H}_{4}$
4. $3-\mathrm{NO}_{2} \mathrm{C}_{6} \mathrm{H}_{4}$
10. $4-\mathrm{FC}_{6} \mathrm{H}_{4}$
5. $2-\mathrm{NO}_{2} \mathrm{C}_{6} \mathrm{H}_{4}$
11. $4-\mathrm{BrC}_{6} \mathrm{H}_{4}$
6. $2-\mathrm{ClC}_{6} \mathrm{H}_{4}$

\section{Antimicrobial Activity}

Scheme-1: Synthesis of 4-Nitro Aniline derived Pyrazolines

Bacterial and fungal strains collected from IMTECH, Chandigarh, India. Antimicrobial studies for pyrazoline derivatives performed by serial dilution method. ${ }^{18}$ The test organisms used were (Bacillus subtilis, Staphylococcus aureus, Escherichia coli, E. faecalis, Pseudomonas fluorescence, Pseudomonas aeruginosa, Klebsiella and Salmonella typhi) and the fungal cultures of (Aspergillus niger, Aspergillus terreus and Trichoderma harzanium). A stock solution of $100 \mu \mathrm{g} / \mathrm{mL}$ of the tested compounds was serially diluted to get the concentration of $50 \mu \mathrm{g} / \mathrm{mL}$ and inoculated with $100 \mu \mathrm{L}$ of suspension of the respective organism in sterile saline. DMSO was used as a solvent. All the inoculated test tubes of synthesized compounds and the standard drugs ciprofloxacin, Ketoconazole, Fluconazole and Tetracycline incubated for $24 \mathrm{~h}$ at $37^{\circ} \mathrm{C}$ and the results were recorded as minimum inhibition concentration (MIC).

\section{General Procedure for the Synthesis of p-Nitroazidobenzene ${ }^{19}$ (1)}

p-nitro aniline $(0.138 \mathrm{~g}, 1 \mathrm{~m} \mathrm{~mol})$ was dissolved in $1: 1 \mathrm{HCl}$ taken in a round bottom flask equipped with stirrer and an ice bath to maintain the temperature between $0-5^{\circ} \mathrm{C}$. Sodium nitrite $(0.069 \mathrm{~g}, 1 \mathrm{mmol})$ dissolved in $25 \mathrm{~mL}$ of ice-cold water was added in portions to the flask with constant stirring followed by $(0.065 \mathrm{~g}, 1 \mathrm{mmol})$ cooled sodium azide solution. The pale yellow color precipitate was washed with sodium carbonate solution and water until the filtrate was neutral to litmus paper and dried. The yield was $98 \%$.

1-Azido-4-nitrobenzene (yellow colored powder) (1): $\mathrm{MP} 80^{\circ} \mathrm{C}$; IR $\left(\mathrm{KBr}, \mathrm{cm}^{-1}\right): 3110,15932127 ;{ }^{1} \mathrm{H}$ NMR (DMSO-d $\left.{ }_{6}, 300 \mathrm{MHz}\right): \delta 7.38(2 \mathrm{H}, \mathrm{d}, \mathrm{J}=3 \mathrm{~Hz}, \mathrm{Ar}), 8.24\left(2 \mathrm{H}, \mathrm{d}, \mathrm{J}=3 \mathrm{~Hz}\right.$ Ar) $;{ }^{13} \mathrm{C}$ NMR $\left(\right.$ DMSO-d $_{6}, 300$ MHz) 147.12, 144.50, 125.96, 120.51; MS: $m / z 164\left[\mathrm{M}^{+}\right]$.

General Procedure for the Synthesis of 1-(4-methyl-1-(4-nitrophenyl)-1H-1, 2, 3-triazole-5-yl) ethanone $^{20}(2)$

p-nitroazidobenzene $(0.164 \mathrm{~g}, 1 \mathrm{mmol})$ was mixed with acetylacetone $(1 \mathrm{~g}, 1 \mathrm{mmol})$ and $\mathrm{K}_{2} \mathrm{CO}_{3}$ $(0.414 \mathrm{~g}, 3 \mathrm{mmol})$ in $20 \mathrm{~mL}$ of $(95 \%)$ ethanol taken in a round bottom flask equipped with a stirrer. The reaction mixture was heated at $80^{\circ} \mathrm{C}$ for 6 hours with constant stirring and the reaction was monitored by TLC. After completion of the reaction, the excess solvent was removed under vacuum and quenched in ice, neutralized with $10 \% \mathrm{HCl}$ and washed with water until the filtrate was neutral to litmus paper and dried. The yield was $94 \%$. 
RASĀYAN J. Chem.

Vol. 13 | No. 1 |676-683| January - March | 2020

Yellow Colored Amorphous Powder (2): MP $147^{\circ} \mathrm{C}$; IR ( $\left.\mathrm{KBr}, \mathrm{cm}^{-1}\right): 3078,1558,1521,1680 ;{ }^{1} \mathrm{H}$ NMR (DMSO-d $6,300 \mathrm{MHz}): 7.97(2 \mathrm{H}, \mathrm{d}, \mathrm{J}=3 \mathrm{~Hz} \mathrm{Ar}), 8.47(2 \mathrm{H}, \mathrm{d}, \mathrm{J}=3 \mathrm{~Hz} \mathrm{Ar}), 2.66\left(3 \mathrm{H}, \mathrm{s}, \mathrm{COCH}_{3}\right), 2.602(3 \mathrm{H}$, $\left.\mathrm{s}, \mathrm{CH}_{3}\right) ;{ }^{13} \mathrm{C}$ NMR (DMSO-d $\left.6,300 \mathrm{MHz}\right) 193.71,148.34,143.55,140.26,138.64,126.91,125.55,40.81$, 39.97, 28.11, 10.26; MS: m/z $246.1\left[\mathrm{M}^{+}\right]$.

General Procedure for 1-(4-methyl)-1-(4-nitrophenyl-1H-1,2,3-triazole-5-yl)-3-phenylprop-2-en-1one $^{21}(1)$

1-(4-methyl-1-(4-nitrophenyl)-1H-1, 2, 3-triazole-5-yl) ethanone $(0.246 \mathrm{~g}, 1 \mathrm{mmol})$ mixed with substituted aromatic aldehydes, $10 \mathrm{~mL}$ of ethanol, $20 \mathrm{~mL}$ of $25 \% \mathrm{NaOH}$ were added and was stirred for $5 \mathrm{~h}$ at room temperature. The completion of the reaction was identified with the help of TLC analysis and the content in the flask was neutralized with $10 \% \mathrm{HCl}$ and washed with water and dried. Yield was $98 \%$.

1. 1-(4-methyl) -1-(4-nitrophenyl-1H- 1, 2, 3-triazole -5-yl)-3-phenylprop-2-en-1-one (Colorless, Amorphous Solid)

MP $207^{\circ} \mathrm{C}$; IR (KBr, cm $\left.{ }^{-1}\right): 3089,1656,1593,1519,1342 ; .{ }^{1} \mathrm{H}$ NMR (DMSO-d 6 , $\left.300 \mathrm{MHz}\right): 8.52(2 \mathrm{H}$, $\mathrm{d}, \mathrm{J}=8.5 \mathrm{~Hz}, \mathrm{Ar}), 8.04(2 \mathrm{H}, \mathrm{d}, \mathrm{J}=9.5 \mathrm{~Hz} \mathrm{Ar}), 7.92(1 \mathrm{H}, \mathrm{d}, \mathrm{J}=9.0 \mathrm{~Hz} \mathrm{H \alpha}), 8.0\left(1 \mathrm{H}, \mathrm{d}, \mathrm{J}=9.0 \mathrm{~Hz} \mathrm{H}_{\beta}\right), 2.69$ $\left(3 \mathrm{H}, \mathrm{s}, \mathrm{CH}_{3}\right), 7.5-7.84(5 \mathrm{H}, \mathrm{m}, \mathrm{Ar}):{ }^{13} \mathrm{C}$ NMR (DMSO-d $\left.6,300 \mathrm{MHz}\right): 183.75,148.43,143.90,143.80$, $140.31,140.0,134.78,131.38,129.59,129.25,127.05,125.91,125.60,123.09,10.52 ; \mathrm{MS}: \mathrm{m} / \mathrm{z}$ $335.09\left[\mathrm{M}^{+}\right]$.

2. 3-(4-methoxyphenyl)-1-(4-methyl-1-(4-nitrophenyl)-1H-1,2,3-triazol-5-yl)prop-2-en-1-one (Pale Yellow Crystalline Solid)

MP $\left.212^{\circ} \mathrm{C} ; \operatorname{IR}\left(\mathrm{KBr}, \mathrm{cm}^{-1}\right): 3078,1680\right), 1597,1520,1343 ;{ }^{1} \mathrm{H}$ NMR(DMSO-d $\left.\mathrm{d}_{6}, 300 \mathrm{MHz}\right): 8.52(2 \mathrm{H}, \mathrm{d}$, $\mathrm{J}=8.5 \mathrm{~Hz} \mathrm{Ar}), 8.04(2 \mathrm{H}, \mathrm{d}, \mathrm{J}=9.5 \mathrm{~Hz} \mathrm{Ar}), 7.94(1 \mathrm{H}, \mathrm{d}, \mathrm{J}=15.6 \mathrm{~Hz} \mathrm{H \alpha}), 7.87\left(1 \mathrm{H}, \mathrm{d}, \mathrm{J}=9.0 \mathrm{~Hz}, \mathrm{H}_{\beta}\right), 7.06$ $(2 \mathrm{H}, \mathrm{d}, \mathrm{J}=8.7 \mathrm{~Hz} \mathrm{Ar}), 7.84(2 \mathrm{H}, \mathrm{d}, \mathrm{J}=8.1 \mathrm{~Hz} \mathrm{Ar}), 2.68\left(3 \mathrm{H}, \mathrm{s}, \mathrm{CH}_{3}\right), 3.84\left(3 \mathrm{H}, \mathrm{s},-\mathrm{OCH}_{3}\right):{ }^{13} \mathrm{C} \mathrm{NMR}$ (DMSO-d 6,300 MHz):183.71, 162.07, 148.43, 143.88, 140.38, 139.74, 131.21, 127.42, 127.07,

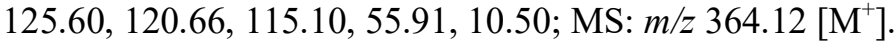

3. 1-(4-methyl-1-(4-nitrophenyl)-1H-1,2,3-triazol-5-yl)-3-(4-nitrophenyl)prop-2-en-1-one, (Brown Amorphous Solid)

MP $218^{\circ} \mathrm{C}$; IR( $\left.\mathrm{KBr}, \mathrm{cm}^{-1}\right): 3082,166,1601,1523,1346 ;{ }^{1} \mathrm{H}$ NMR(DMSO-d $\left.\mathrm{d}_{6}, 300 \mathrm{MHz}\right): 8.38$ ( $2 \mathrm{H}, \mathrm{d}$, $\left.\mathrm{J}=8.1 \mathrm{~Hz} \mathrm{Ar}) 7.94(2 \mathrm{H}, \mathrm{d}, \mathrm{J}=9 \mathrm{~Hz} \mathrm{Ar}), 8.04(1 \mathrm{H}, \mathrm{d}, \mathrm{J}=8.8 \mathrm{~Hz} \mathrm{H \alpha}), 8.38(1 \mathrm{H}, \mathrm{d}, \mathrm{J}=8.8 \mathrm{~Hz} \mathrm{H})_{\beta}\right), 8.17(2 \mathrm{H}$, $\mathrm{d}, \mathrm{J}=2.8 \mathrm{~Hz} \mathrm{Ar}), 8.11(2 \mathrm{H}, \mathrm{d}, \mathrm{J}=2.8 \mathrm{~Hz} \mathrm{dAr}), 2.66\left(3 \mathrm{H}, \mathrm{s}, \mathrm{CH}_{3}\right) ;{ }^{13} \mathrm{C}$ NMR(DMSO-d $\left.\mathrm{d}_{6}, 300 \mathrm{MHz}\right)$ : $182.40_{\mathrm{C}=\mathrm{O}}, 163.30,151,149,143,143,142,135,134,125,123.3,117,10.43_{\mathrm{CH} 3} . \mathrm{MS}: \mathrm{m} / \mathrm{z} 378.77$ $\left[\mathrm{M}^{+}\right]$.

4. 1-(4-methyl-1-(4-nitrophenyl)-1H-1,2,3-triazol-5-yl)-3-(3-nitrophenyl)prop-2-en-1-one (Brown Amorphous Solid)

MP195 ${ }^{\circ} \mathrm{C} ; \operatorname{IR}\left(\mathrm{KBr}, \mathrm{cm}^{-1}\right): 3091,1667,1598,1521,1346 ;{ }^{1} \mathrm{HNMR}\left(\mathrm{DMSO}-\mathrm{d}_{6}, 300 \mathrm{MHz}\right): 8.53(2 \mathrm{H}, \mathrm{d}$, $\mathrm{J}=9.0 \mathrm{~Hz} \mathrm{Ar}), 8.33(2 \mathrm{H}, \mathrm{d}, \mathrm{J}=9.0 \mathrm{~Hz} \mathrm{Ar}), 8.05(1 \mathrm{H}, \mathrm{d}, \mathrm{J}=6.6 \mathrm{~Hz} \mathrm{H \alpha}), 7.78\left(1 \mathrm{H}, \mathrm{d}, \mathrm{J}=7.8 \mathrm{H}_{\beta}\right), 7.997-$ $8.147(4 \mathrm{H}, \mathrm{m}, \mathrm{Ar}), 2.66\left(3 \mathrm{H}, \mathrm{s}, \mathrm{CH}_{3}\right) ;{ }^{13} \mathrm{C}$ NMR (DMSO-d $\left.6,300 \mathrm{MHz}\right): 183.42,150,148,148.37,144$, $143.65,141,140,136.57,134,126.92,126.73,124,123,118,10.53$; MS: $m / z 378.77\left[\mathrm{M}^{+}\right]$.

5. 1-(4-methyl-1-(4-nitrophenyl)-1H-1,2,3-triazol-5-yl)-3-(2-nitrophenyl)prop-2-en-1-one (Brown Amorphous Solid)

MP $208^{\circ} \mathrm{C} ; \mathrm{IR}\left(\mathrm{KBr}, \mathrm{cm}^{-1}\right): 3082,168,1602,1527,1348 ;{ }^{1} \mathrm{HNMR}\left(\mathrm{DMSO}-\mathrm{d}_{6}, 300 \mathrm{MHz}\right): 8.53(2 \mathrm{H}, \mathrm{d}$, $\mathrm{J}=8.7 \mathrm{~Hz} \mathrm{Ar}), 8.18(2 \mathrm{H}, \mathrm{d}, \mathrm{J}=9.0 \mathrm{~Hz} \mathrm{Ar}), 8.13(1 \mathrm{H} \mathrm{d}, \mathrm{J}=10.5 \mathrm{~Hz} \mathrm{H \alpha}),, 8.02\left(1 \mathrm{H}, \mathrm{d}, \mathrm{J}=9.3 \mathrm{~Hz} \mathrm{H} \mathrm{H}_{\beta}\right), 7.88$ $(1 \mathrm{H}, \mathrm{t}, \mathrm{J}=7.5 \mathrm{~Hz}, 7.2 \mathrm{~Hz} \mathrm{Ar}), 7.77(1 \mathrm{H}, \mathrm{t}, \mathrm{J}=7.8 \mathrm{~Hz}, 7.5 \mathrm{~Hz} \mathrm{Ar}), 2.70\left(3 \mathrm{H}, \mathrm{s}, \mathrm{CH}_{3}\right):{ }^{13} \mathrm{C}$ NMR $\left(\mathrm{DMSO}_{6}\right.$, $300 \mathrm{MHz}): 183.37,163.5,149.25,148.50,143.56,140.56,140.23,138.87,134.56,130.10,129.81$,

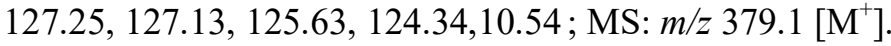

6. 3-(2-chlorophenyl)-1-(4-methyl-1-(4-nitrophenyl)-1H-1,2,3-triazol-5-yl)prop-2-en-1-one (Colorless Solid)

MP $202^{\circ} \mathrm{C} ; \mathrm{IR}\left(\mathrm{KBr}, \mathrm{cm}^{-1}\right): 3091\left(\mathrm{C}-\mathrm{H}\right.$ str):1683,1595), 1525,1344; ${ }^{1} \mathrm{HNMR}\left(\mathrm{DMSO}-\mathrm{d}_{6}, 300 \mathrm{MHz}\right): 8.49$ 
$(2 \mathrm{H}, \mathrm{d}, \mathrm{J}=9.0 \mathrm{~Hz} \mathrm{Ar}), 8.32(2 \mathrm{H}, \mathrm{d}, \mathrm{J}=9.0 \mathrm{~Hz} \mathrm{Ar}), 7.63(1 \mathrm{H}, \mathrm{d}, \mathrm{J}=9.0 \mathrm{~Hz} \mathrm{H \alpha}), 7.72\left(1 \mathrm{H}, \mathrm{d}, \mathrm{J}=9.0 \mathrm{~Hz} \mathrm{H}_{\beta}\right)$, 7.5- 8.5 (4H, (m, Ar), 2.69 (3H, s, $\left.\mathrm{CH}_{3}\right):{ }^{13} \mathrm{C}$ NMR (DMSO-d $\left.6,300 \mathrm{MHz}\right): 183.49,148,144,143,141$, $143.80,140.63,140.33,140.20,138.68,138.60,134.94,132.98,129.59,127.05,125.91,123.09$, 10.5; MS: $m / z$ 368.1[$\left[\mathrm{M}^{+}\right], 370.1(\mathrm{M}+2](1: 3$ ratio.

7. 3-(3-chlorophenyl)-1-(4-methyl-1-(4-nitrophenyl)-1H-1,2,3-triazol-5-yl)prop-2-en-1-one (Pale Yellow Solid)

MP $203^{\circ} \mathrm{C}$; IR( $\left.\mathrm{KBr}, \mathrm{cm}^{-1}\right): 3091\left(\mathrm{C}-\mathrm{H}\right.$ str):3078, 169, 1596), 1515, 1342; ${ }^{1} \mathrm{HNMR}\left(\mathrm{DMSO}-\mathrm{d}_{6}, 300 \mathrm{MHz}\right)$ : $8.51(2 \mathrm{H}, \mathrm{d}, \mathrm{J}=9.0 \mathrm{~Hz} \mathrm{Ar}), 7.78(2 \mathrm{H}, \mathrm{d}, \mathrm{J}=9.0 \mathrm{~Hz} \mathrm{Ar}), 8.1(1 \mathrm{H}, \mathrm{d}, \mathrm{J}=16.2 \mathrm{~Hz} \mathrm{H \alpha}), 7.89(1 \mathrm{H}, \mathrm{d}, \mathrm{J}=16.2 \mathrm{~Hz}$ $\mathrm{H}_{\beta}$ ), 7.4-7.7 (4H, m, Ar), $2.78\left(3 \mathrm{H}, \mathrm{s}, \mathrm{CH}_{3}\right):{ }^{13} \mathrm{C}$ NMR (DMSO-d $\left.6.300 \mathrm{MHz}\right): 183.4,142.04,136.25$, $130.18,129.94,127.96,126.70,125.63,124.91,123.61,10.21$; MS: $m / z 368.1\left[\mathrm{M}^{+}\right], 370.1[\mathrm{M}+2]$ (1:3ratio).

8. 3-(4-chlorophenyl)-1-(4-methyl-1-(4-nitrophenyl)-1H-1,2,3-triazol-5-yl)prop-2-en-1-one, (Colorless Amorphous Solid)

MP189 ${ }^{\circ}$; IR( $\left.\mathrm{KBr}, \mathrm{cm}^{-1}\right): 2966,1680,1597,1525,1344 ;{ }^{1} \mathrm{HNMR}\left(\mathrm{DMSO}_{6}, \mathrm{~d}_{6}, 300 \mathrm{MHz}\right): 8.58 \quad 2 \mathrm{H},(\mathrm{d}$, $\mathrm{J}=5.7 \mathrm{~Hz}$ Ar), $8.03(2 \mathrm{H}, \mathrm{d}, \mathrm{J}=5.4 \mathrm{~Hz} \mathrm{Ar}), 8.1 \quad(1 \mathrm{H}, \mathrm{d}, \mathrm{J}=10.5 \mathrm{~Hz} \mathrm{H \alpha}), 7.97\left(1 \mathrm{H}, \mathrm{d}, \mathrm{J}=8.1 \mathrm{~Hz} \mathrm{H} \mathrm{H}_{\beta}\right), 7.62$ $(2 \mathrm{H}, \mathrm{d}, \mathrm{J}=5.3 \mathrm{~Hz} \mathrm{Ar}), 8.03 \quad(2 \mathrm{H}, \mathrm{d}, \mathrm{J}=5.2 \mathrm{~Hz} \mathrm{Ar}), 2.71\left(3 \mathrm{H}, \mathrm{s},\left(\mathrm{CH}_{3}\right):{ }^{13} \mathrm{C}\right.$ NMR (DMSO-d 6,300 MHz):183.66, 167, 148, 143, 140.2, 140, 135, 133, 131, 130, 128.64, 129, 125, 123.80, 123.0, 10.52; MS: $m / z 368.1\left[\mathrm{M}^{+}\right], 371.18[\mathrm{M}+2](1: 3$ ratio).

9. 3-(2-hydroxyphenyl)-1-(4-methyl-1-(4-nitrophenyl)-1H-1,2,3-triazol-5-yl)prop-2-en-1-one, (Pale Yellow Amorphous Solid)

MP $209^{\circ} \mathrm{C} ; \mathrm{IR}\left(\mathrm{KBr}, \mathrm{cm}^{-1}\right): 2885,1681,1575,1523,1344 ;{ }^{1} \mathrm{HNMR}\left(\mathrm{DMSO}-\mathrm{d}_{6}, 300 \mathrm{MHz}\right): 8.58(2 \mathrm{H}, \mathrm{d}$, $\mathrm{J}=9.0 \mathrm{~Hz} \mathrm{Ar}), 8.10(2 \mathrm{H}, \mathrm{d}, \mathrm{J}=9.0 \mathrm{~Hz} \mathrm{Ar}), 7.77(1 \mathrm{H}, \mathrm{d}, \mathrm{J}=7.8 \mathrm{~Hz} \mathrm{H \alpha}), 6.94(1 \mathrm{H}, \mathrm{d}, \mathrm{J}=10.5 \mathrm{~Hz} \mathrm{H})_{\beta}, 7.02-$ 7.3 (4H, m, Ar), $2.74\left(3 \mathrm{H}, \mathrm{s}, \mathrm{CH}_{3}\right):{ }^{13} \mathrm{C}$ NMR (DMSO-d $\left.6,300 \mathrm{MHz}\right): 183.71,162.07,148.43,143.88$, $140.38,139.74,131.21,127.42,127.07,125.60,120.66,115.00,10.50$; MS: $m / z 350.1\left[\mathrm{M}^{+}\right]$.

10. 3-(4-fluorophenyl)-1-(4-methyl-1-(4-nitrophenyl)-1H-1,2,3-triazol-5-yl)prop-2-en-1-one (Colorless Crystalline Solid)

MP $\left.206^{\circ} \mathrm{C} ; \operatorname{IR}\left(\mathrm{KBr}, \mathrm{cm}^{-1}\right): 3094,1668,1597\right), 1523,1343 ;{ }^{1} \mathrm{HNMR}\left(\mathrm{DMSO}-\mathrm{d}_{6}, 300 \mathrm{MHz}\right): 8.52(2 \mathrm{H}, \mathrm{d}$, $\mathrm{J}=9.0 \mathrm{~Hz} \mathrm{Ar}), 7.36(2 \mathrm{H}, \mathrm{d}, \mathrm{J}=8.7 \mathrm{~Hz} \mathrm{Ar}), 8.04(2 \mathrm{H}, \mathrm{d}, \mathrm{J}=4.2 \mathrm{~Hz} \mathrm{Ar}), 7.93(2 \mathrm{H}, \mathrm{d}, \mathrm{J}=6.0 \mathrm{~Hz}, \mathrm{Ar}), 7.91$ $(1 \mathrm{H}, \mathrm{d}, \mathrm{J}=16.2 \mathrm{~Hz}, \mathrm{H \alpha}), 8.04\left(1 \mathrm{H}, \mathrm{d}, \mathrm{J}=11.4 \mathrm{~Hz} \mathrm{H}_{\beta}\right), 2.68\left(3 \mathrm{H}, \mathrm{s}, \mathrm{CH}_{3}\right):{ }^{13} \mathrm{C}$ NMR $($ DMSO-d, 300 MHz): 183.71, 165.6, 162.4, 148.4, 143.7, 142.7, 140.3, 140, 131.75, 131.6, 131.00, 127.07, 125.60, 122.07, 116.7, 116.40, 10.52; MS: $m / z 352.1\left[\mathrm{M}^{+}\right]$.

11. 3-(4-bromophenyl)-1-(4-methyl-1-(4-nitrophenyl)-1H-1,2,3-triazol-5-yl)prop-2-en-1-one (Pale Yellow Solid)

MP $192^{\circ} \mathrm{C} ; \operatorname{IR}\left(\mathrm{KBr}, \mathrm{cm}^{-1}\right): 3090,1681,1587,1527,1345 ;{ }^{1} \mathrm{HNMR}\left(\mathrm{DMSO}-\mathrm{d}_{6}, 300 \mathrm{MHz}\right): 8.52(2 \mathrm{H}, \mathrm{d}$, $\mathrm{J}=9.0 \mathrm{~Hz} \mathrm{Ar}), 7.70(2 \mathrm{H}, \mathrm{d}, \mathrm{J}=8.4 \mathrm{~Hz} \mathrm{Ar}), 8.08(1 \mathrm{H}, \mathrm{J}=13.2 \mathrm{~Hz} \mathrm{H \alpha}), 7.82(1 \mathrm{H}, \mathrm{d}, \mathrm{J}=26.4 \mathrm{~Hz} \mathrm{H})_{\beta}, 8.03$ $(2 \mathrm{H}, \mathrm{d}, \mathrm{J}=6.3 \mathrm{~Hz} \mathrm{Ar}), 7.88(2 \mathrm{H}, \mathrm{d}, \mathrm{J}=8.4 \mathrm{~Hz} \mathrm{Ar}), 2.68\left(3 \mathrm{H}, \mathrm{s}, \mathrm{CH}_{3}\right):{ }^{13} \mathrm{C}$ NMR (DMSO-d $\left.\mathrm{d}_{6}, 300 \mathrm{MHz}\right)$ : 183.60, 167.08, 148.45, 143, 140.29, 140, 134.9, 136.5, 131.7, 131.1, 130.5, 127.35, 125.61, 123.8,

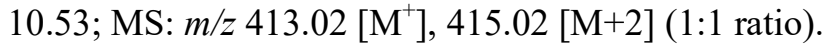

General procedure for the synthesis of 4-Methyl-1-(4-nitrophenyl)-5-(5-phenyl-4, 5-dihydro-1Hpyrazole-3-yl)-1H-1, 2, 3 triazole (4)

1(4-methyl) -1-(4-nitrophenyl-1H1，2，3-triazole -5-yl)-3-phenylprop-2-en-1-one (0.334g, 1mmol), hydrazine hydrate $(0.75 \mathrm{~g}, 1.5 \mathrm{mmol}), 20 \mathrm{~mL}$ of ethanol, $5 \mathrm{~mL}$ of $20 \%$ glacial acetic acid were mixed and heated with stirring for 8 hours at $80^{\circ} \mathrm{C}$. The content in the flask was neutralized with $\mathrm{Na}_{2} \mathrm{CO}_{3}$ and washed with water and dried. The yield obtained was $85 \%$.

1. 4-Methyl-1-(4-nitrophenyl)-5-(5-phenyl-4,5-dihydro-1H-pyrazole-3-yl)-1H-1,2,3-triazole (Yellow Colour Solid)

MP $208^{\circ} \mathrm{C}$; IR( $\left.\mathrm{KBr}, \mathrm{cm}^{-1}\right): 3327,3089,1609,1519,1343 ;{ }^{1} \mathrm{HNMR}\left(\mathrm{DMSO}-\mathrm{d}_{6}, 300 \mathrm{MHz}\right): 8.46(2 \mathrm{H}, \mathrm{d}$, $\mathrm{J}=9.0 \mathrm{~Hz} \mathrm{Ar}), 8.01(2 \mathrm{H}, \mathrm{d}, \mathrm{J}=9.0 \mathrm{~Hz} \mathrm{Ar}), 3.01(1 \mathrm{H}, \mathrm{dd}, \mathrm{J}=10.2 \mathrm{~Hz}, 10.4 \mathrm{~Hz} \mathrm{C}-\mathrm{H}), 3.04(1 \mathrm{H}, \mathrm{dd}$, 679 


\section{RASĀYAN J. Chem. \\ Vol. 13 | No. 1 |676-683| January - March | 2020}

$\mathrm{J}=10.5 \mathrm{~Hz}, 10.8 \mathrm{~Hz} \mathrm{C}-\mathrm{H}), 4.86(1 \mathrm{H}, \mathrm{t}, \mathrm{J}=10.5 \mathrm{~Hz} \mathrm{C}-\mathrm{H}), 7.402(1 \mathrm{H}, \mathrm{s}, \mathrm{N}-\mathrm{H}), 7.2$ to $7.5(5 \mathrm{H}, \mathrm{m}, \mathrm{Ar}), 2.62$ $3 \mathrm{H},\left(\mathrm{s}, \mathrm{CH}_{3}\right):{ }^{13} \mathrm{C}$ NMR (DMSO-d $\left.6,300 \mathrm{MHz}\right): 147.98,144.05,143.09,140.98,139.98,132.54$, $128.91,127.68,127.08,126.36,125.54,63.09_{\mathrm{C}-\mathrm{H}}, 42.40,10.58$; MS: $m / z 348.13\left[\mathrm{M}^{+}\right]$.

2. 5-(5-(4-methoxyphenyl)-4,5-dihydro-1H-pyrazol-3-yl)-4-methyl-1-(4-nitrophenyl)-1H-1, 2 3triazole (Pale Yellow Amorphous Solid)

MP $210^{\circ} \mathrm{C}$; IR( $\left.\mathrm{KBr}, \mathrm{cm}^{-1}\right): 3327,3089,1610,1519,1342 ;{ }^{1} \mathrm{HNMR}\left(\mathrm{DMSO}-\mathrm{d}_{6}, 300 \mathrm{MHz}\right): 8.48$ (2H, d, $\mathrm{J}=8.5 \mathrm{~Hz} \mathrm{Ar}), 8.00(2 \mathrm{H}, \mathrm{d}, \mathrm{J}=8.5 \mathrm{~Hz} \mathrm{Ar}), 3.00\left(1 \mathrm{H}, \mathrm{dd}, \mathrm{J}=10.5 \mathrm{~Hz}, 10.5 \mathrm{~Hz} \mathrm{C}-\mathrm{H}_{2 \mathrm{a}}\right), 3.59(1 \mathrm{H}, \mathrm{dd}$, $\left.\mathrm{J}=10.5 \mathrm{~Hz}, 10.8 \mathrm{~Hz} \mathrm{C}-\mathrm{H}_{2 \mathrm{~b}}\right), 4.83(1 \mathrm{H}, \mathrm{t}, \mathrm{J}=10.5 \mathrm{~Hz} \mathrm{C}-\mathrm{H}), 7.63(1 \mathrm{H}, \mathrm{s}, \mathrm{N}-\mathrm{H}), 6.93(2 \mathrm{H}, \mathrm{d}, \mathrm{J}=8.0 \mathrm{~Hz} \mathrm{Ar})$, $7.33(2 \mathrm{H}, \mathrm{d}, \mathrm{J}=8.0 \mathrm{~Hz} \mathrm{Ar}), 2.62\left(3 \mathrm{H}, \mathrm{s}, \mathrm{CH}_{3}\right), 3.75\left(3 \mathrm{H}, \mathrm{s}, \mathrm{OCH}_{3}\right):{ }^{13} \mathrm{C}$ NMR $\left(\mathrm{DMSO}_{6} \mathrm{~d}_{6}, 300 \mathrm{MHz}\right)$ : $148.12,145.7,145.2,141.5,136.12,135.05,134.1,129.9,129.2,127.05,125.1,124.9,65.9_{\text {C-H }}, 44.4$, 11.9; MS: $m / z 378.14\left[\mathrm{M}^{+}\right]$.

3. 4-methyl-1-(4-nitrophenyl)-5-(5-(4-nitrophenyl)-4,5-dihydro-1H-pyrazol-3-yl)-1H-1,2,3-triazole (Yellow Color Amorphous Solid)

MP $211^{\circ} \mathrm{C}$; IR( $\left.\mathrm{KBr}, \mathrm{cm}^{-1}\right): 3317,3087,1599,1517,1345 ;{ }^{1} \mathrm{HNMR}\left(\mathrm{DMSO}-\mathrm{d}_{6}, 300 \mathrm{MHz}\right): 8.48$ (2H, d, $\mathrm{J}=9.2 \mathrm{~Hz}$ Ar $), 8.0(2 \mathrm{H}, \mathrm{d}, \mathrm{J}=8.8 \mathrm{~Hz} \mathrm{Ar}), 3.04\left(1 \mathrm{H}, \mathrm{dd}, \mathrm{J}=10.4 \mathrm{~Hz}, 10.5 \mathrm{~Hz} \mathrm{C}-\mathrm{H}_{2 \mathrm{a}}\right), 3.76(1 \mathrm{H}, \mathrm{dd}$, $\left.\mathrm{J}=10.2 \mathrm{~Hz}, 10.5 \mathrm{~Hz} \mathrm{C}-\mathrm{H}_{2 \mathrm{~b}}\right), 5.04(1 \mathrm{H}, \mathrm{t}, \mathrm{J}=7.6 \mathrm{~Hz} \mathrm{C}-\mathrm{H}), 7.98(1 \mathrm{H}, \mathrm{s}, \mathrm{N}-\mathrm{H}), 8.25(2 \mathrm{H}, \mathrm{d}, \mathrm{J}=8.4 \mathrm{~Hz} \mathrm{Ar})$, $7.70(2 \mathrm{H}, \mathrm{d}, \mathrm{J}=8.8 \mathrm{~Hz} \mathrm{Ar}), 2.62\left(3 \mathrm{H}, \mathrm{s}, \mathrm{CH}_{3}\right):{ }^{13} \mathrm{C}$ NMR (DMSO-d $\left.6,300 \mathrm{MHz}\right): 151.2,148,147.1$, 143.7, 140.9, 139.0, 132.0, 128.4, 126.4, 125.5, 124.1, 62.35, 42.45, 10.56; MS: $m / z 393.12\left[\mathrm{M}^{+}\right]$.

4. 4-methyl-1-(4-nitrophenyl)-5-(5-(3-nitrophenyl)-4,5-dihydro-1H-pyrazol-3-yl)-1H-1,2,3-triazole (Yellow Colored Amorphous Solid)

MP $211^{\circ} \mathrm{C}$; IR( $\left.\mathrm{KBr}, \mathrm{cm}^{-1}\right): 3284,3112,1690,1516,1345 ;{ }^{1} \mathrm{HNMR}\left(\mathrm{DMSO}-\mathrm{d}_{6}, 300 \mathrm{MHz}\right): 8.23(2 \mathrm{H}, \mathrm{d}$, $\mathrm{J}=9.0 \mathrm{~Hz} \mathrm{Ar}), 7.78(2 \mathrm{H}, \mathrm{d}, \mathrm{J}=7.8 \mathrm{~Hz} \mathrm{Ar}), 3.73\left(1 \mathrm{H}, \mathrm{dd}, \mathrm{J}=10.2 \mathrm{~Hz}, 10.5 \mathrm{~Hz} \mathrm{C}-\mathrm{H}_{2 \mathrm{a}}\right), 3.01(1 \mathrm{H}, \mathrm{dd}$, $\left.\mathrm{J}=10.5 \mathrm{~Hz}, 10.8 \mathrm{~Hz} \mathrm{C}-\mathrm{H}_{2 \mathrm{~b}}\right), 5.04(1 \mathrm{H}, \mathrm{t}, \mathrm{J}=10.5 \mathrm{~Hz} \mathrm{C}-\mathrm{H}), 8.81(1 \mathrm{H}, \mathrm{s}, \mathrm{N}-\mathrm{H}), 7.90(2 \mathrm{H}, \mathrm{d}, \mathrm{J}=8.1 \mathrm{~Hz} \mathrm{Ar})$, $8.01(2 \mathrm{H}, \mathrm{d}, \mathrm{J}=9.0 \mathrm{~Hz} \mathrm{Ar}), 2.62\left(3 \mathrm{H}, \mathrm{s}, \mathrm{CH}_{3}\right):{ }^{13} \mathrm{C}$ NMR (DMSO-d $\left.6,300 \mathrm{MHz}\right): 167.20,166.61$, $150.77,148.30,147.98,145.70,140.0,139.0,134.0,132.0,130.0,129.05,125.91,123.09,62.16$, 42.4, 10.56 ;MS: $m / z 393.12\left[\mathrm{M}^{+}\right]$.

5. 4-methyl-1-(4-nitrophenyl)-5-(5-(2-nitrophenyl)-4,5-dihydro-1H-pyrazol-3-yl)-1H-1,2,3-triazole (Yellow Color Amorphous Solid)

MP. $210^{\circ} \mathrm{C}$; IR $\left(\mathrm{KBr}, \mathrm{cm}^{-1}\right): 3317,3087,1599,1518,1345:{ }^{1} \mathrm{HNMR}\left(\mathrm{DMSO}-\mathrm{d}_{6}, 300 \mathrm{MHz}\right): 8.27(2 \mathrm{H}, \mathrm{d}$, $\mathrm{J}=8.0 \mathrm{~Hz}$ Ar $), 7.79(2 \mathrm{H}, \mathrm{d}, \mathrm{J}=7.9 \mathrm{z} \mathrm{Ar}), 3.73\left(1 \mathrm{H}, \mathrm{dd}, \mathrm{J}=9.2 \mathrm{~Hz}, 10.8 \mathrm{~Hz} \mathrm{C}-\mathrm{H}_{2 \mathrm{a}}\right), 3.18 \quad(1 \mathrm{H}, \mathrm{dd}$, $\left.\mathrm{J}=10.0 \mathrm{~Hz}, 9.8 \mathrm{~Hz} \mathrm{C}-\mathrm{H}_{2 \mathrm{~b}}\right), 4.80(1 \mathrm{H}, \mathrm{t}, \mathrm{J}=10.1 \mathrm{~Hz} \mathrm{C}-\mathrm{H}), 8.75(1 \mathrm{H}, \mathrm{s}, \mathrm{N}-\mathrm{H}), 8.65-8.7(4 \mathrm{H}, \mathrm{m}, \mathrm{Ar}), 2.62$ $\left(3 \mathrm{H}, \mathrm{s}, \mathrm{CH}_{3}\right):{ }^{13} \mathrm{C}$ NMR (DMSO-d $\left.\mathrm{d}_{6}\right) 167.20,165.6,151.7,149.98,149.30,143.71,142.7,140.8,139.5$,

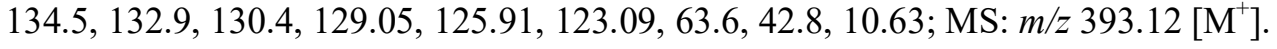

6. 5-(5-(2-chlorophenyl)-4,5-dihydro-1-H-pyrazol-3-yl)-4-methyl-1-(4-nitrophenyl)-1H-1,2,3triazole (Yellow Color Amorphous Solid)

MP $212^{\circ} \mathrm{C} ; \mathrm{IR}\left(\mathrm{KBr}, \mathrm{cm}^{-1}\right): 3325,3078,1599,1523,1345:{ }^{1} \mathrm{HNMR}\left(\mathrm{DMSO}-\mathrm{d}_{6}, 300 \mathrm{MHz}\right): 8.47(2 \mathrm{H}$, $\mathrm{d}, \mathrm{J}=9.0 \mathrm{~Hz} \mathrm{Ar}), 7.77(2 \mathrm{H}, \mathrm{d}, \mathrm{J}=9.0 \mathrm{~Hz} \mathrm{Ar}), 3.25\left(1 \mathrm{H}, \mathrm{dd}, \mathrm{J}=9.3 \mathrm{~Hz}, 9.3 \mathrm{~Hz} \mathrm{C}-\mathrm{H}_{2 \mathrm{a}}\right), 3.87(1 \mathrm{H}, \mathrm{dd}$, $\left.\mathrm{J}=10.8 \mathrm{~Hz}, 2.4 \mathrm{~Hz} \mathrm{C}-\mathrm{H}_{2 \mathrm{~b}}\right), 5.36(1 \mathrm{H}, \mathrm{t}, \mathrm{J}=10.5 \mathrm{~Hz} \mathrm{C}-\mathrm{H}), 6.76(1 \mathrm{H}, \mathrm{s}, \mathrm{N}-\mathrm{H}), 7.4-7.6(4 \mathrm{H}, \mathrm{m}, \mathrm{Ar}), 2.69$ $\left(3 \mathrm{H}, \mathrm{s}, \mathrm{CH}_{3}\right):{ }^{13} \mathrm{C}$ NMR $\left(\mathrm{DMSO}-\mathrm{d}_{6}\right): 147.84,146.05,140.80,139.59,138.75,132.56,131.32,129.77$,

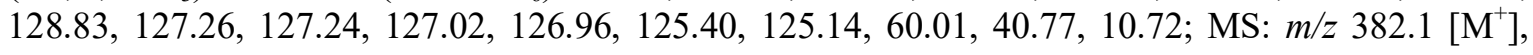
$384.1[\mathrm{M}+2]$.

7. 5-(5-(3-chlorophenyl)-4,5-dihydro-1-H-pyrazol-3-yl)-4-methyl-1-(4-nitrophenyl)-1H-1,2, 3triazole (Yellow Color Amorphous Solid)

MP $215^{\circ} \mathrm{C}$; IR(KBr, $\left.\mathrm{cm}^{-1}\right): 3324,3086,1597,1528,1346 ;{ }^{1} \mathrm{HNMR}\left(\mathrm{DMSO}-\mathrm{d}_{6}, 300 \mathrm{MHz}\right): 8.47$ (2H, d, $\mathrm{J}=9.0 \mathrm{~Hz} \mathrm{Ar}), 7.78(2 \mathrm{H}, \mathrm{d}, \mathrm{J}=8.7 \mathrm{~Hz} \mathrm{Ar}), 3.8\left(1 \mathrm{H}, \mathrm{dd}, \mathrm{J}=7.5 \mathrm{~Hz}, 10.8 \mathrm{~Hz} \mathrm{C}-\mathrm{H}_{2 \mathrm{a}}\right), 3.33(1 \mathrm{H}, \mathrm{dd}, \mathrm{J}=8.7 \mathrm{~Hz}$, 9.0Hz C-H $\left.\mathrm{H}_{2 \mathrm{~b}}\right), 4.91(1 \mathrm{H}, \mathrm{t}, \mathrm{J}=6.3 \mathrm{~Hz} \mathrm{C}-\mathrm{H}), 7.2(4 \mathrm{H}, \mathrm{m}, \mathrm{Ar}), 7.39(1 \mathrm{H}, \mathrm{s}, \mathrm{N}-\mathrm{H}), 2.70\left(3 \mathrm{H}, \mathrm{s}, \mathrm{CH}_{3}\right):{ }^{13} \mathrm{C}$ 680 
NMR (DMSO-d $\left.\mathrm{d}_{6}\right)$ :148.11, 147.99, 145.91, 144.82, 140.32, 139.50, 138.12, 133.86, 131.98, 129.63, $128,127.26,127.24,127.02,126.96,125.40,125.14,62.27,41.98,10.16$; MS: $m / z 382.1\left[\mathrm{M}^{\dagger}\right], 384.1$ $[\mathrm{M}+2]$.

8. 5-(5-(4-chlorophenyl)-4,5-dihydro-1-H-pyrazol-3-yl)-4-methyl-1-(4-nitrophenyl)-1H-1,2,3 triazole(Yellow Color Amorphous Solid)

MP $213^{\circ} \mathrm{C}$; IR( $\left.\mathrm{KBr}, \mathrm{cm}^{-1}\right): 3359,2928,1609,1522,1346 ;{ }^{1} \mathrm{HNMR}\left(\mathrm{DMSO}-\mathrm{d}_{6}, 300 \mathrm{MHz}\right): 7.92$ (2H, d, $\mathrm{J}=8.7 \mathrm{~Hz} \mathrm{Ar}), 7.58(2 \mathrm{H}, \mathrm{d}, \mathrm{J}=8.7 \mathrm{~Hz} \mathrm{Ar}), 8.51(2 \mathrm{H}, \mathrm{d}, \mathrm{J}=3.9 \mathrm{~Hz}) \mathrm{Ar}, 8.49(2 \mathrm{H}, \mathrm{d}, \mathrm{J}=8.4 \mathrm{~Hz} \mathrm{Ar}), 3.09$ $\left(1 \mathrm{H}, \mathrm{dd}, \mathrm{J}=10.1 \mathrm{~Hz}, 9.0 \mathrm{~Hz}\right.$ C- $\left.\mathrm{H}_{2 \mathrm{a}}\right), 3.86\left(1 \mathrm{H}, \mathrm{dd}, \mathrm{J}=10.1 \mathrm{~Hz}, 9.0 \mathrm{~Hz} \mathrm{C}-\mathrm{H}_{2 \mathrm{~b}}\right), 5.09(1 \mathrm{H}, \mathrm{t}, \mathrm{J}=6.3 \mathrm{~Hz} \mathrm{C}-\mathrm{H})$, $7.21(1 \mathrm{H}, \mathrm{s}, \mathrm{N}-\mathrm{H}), 2.66\left(3 \mathrm{H}, \mathrm{s}, \mathrm{CH}_{3}\right):{ }^{13} \mathrm{C}$ NMR (DMSO-d 6 ):148.11, 146.99, 144.91, 143.82, 139.32, 139.0, 137.1, 132.8, 130.9, 127.6, 127, 127.86, 127.24, 126.0, 125.9, 125.4, 124.1, 61.2, 40.9, 11.6; MS: $m / z$ 382.1 [M $], 384.1[\mathrm{M}+2]$.

9. 2-(3-(4-methyl-1-(4-nitrophenyl)-1H-1,2,3-triazol-5-yl)-4,5-dihydro-1H-pyrazol-5-yl) phenol(Yellow Color Amorphous Solid):

MP $203^{\circ} \mathrm{C}$; IR( $\left.\mathrm{KBr}, \mathrm{cm}^{-1}\right): 3337,3091,1626,1520,1346 ;{ }^{1} \mathrm{HNMR}\left(\mathrm{DMSO}-\mathrm{d}_{6}, 300 \mathrm{MHz}\right): 7.19$ (2H, d, $\mathrm{J}=8.4 \mathrm{~Hz}$ Ar), $6.70(2 \mathrm{H}, \mathrm{d}, \mathrm{J}=8.8 \mathrm{~Hz} \mathrm{Ar}), 6.8-7.4(4 \mathrm{H}, \mathrm{m}, \mathrm{Ar}), 2.89\left(1 \mathrm{H}, \mathrm{dd}, \mathrm{J}=10.0 \mathrm{~Hz}, 9.6 \mathrm{~Hz} \mathrm{C}-\mathrm{H}_{2 \mathrm{a}}\right)$, $3.57\left(1 \mathrm{H}, \mathrm{dd}, \mathrm{J}=10.4 \mathrm{~Hz}, 10.4 \mathrm{~Hz} \mathrm{C}-\mathrm{H}_{2 \mathrm{~b}}\right), 4.96(1 \mathrm{H}, \mathrm{t}, \mathrm{J}=10.0 \mathrm{~Hz} \mathrm{C}-\mathrm{H}), 5.59(1 \mathrm{H}, \mathrm{s}, \mathrm{N}-\mathrm{H}), 9.7(1 \mathrm{H}, \mathrm{s}, \mathrm{O}-$ $\mathrm{H}), 2.43\left(3 \mathrm{H}, \mathrm{s}, \mathrm{CH}_{3}\right):{ }^{13} \mathrm{C}$ NMR $\left(\mathrm{DMSO}_{-} \mathrm{d}_{6}\right): 149.2,147.5,146.4,143.9,136.5,131.9,129.2,128.5$, 127.1, 126.9, 61.8 46,.1, 13.2; MS: $m / \mathrm{z} 364.13\left[\mathrm{M}^{+}\right]$.

10. 5-(5-(4-fluorophenyl)-4,5-dihydro-1H-pyrazol-3-yl)-4-methyl-1-(4-nitrophenyl)-1H-1,2,3triazole(Pale Yellow Color Amorphous Solid)

MP $209^{\circ} \mathrm{C}$; IR $\left(\mathrm{KBr}, \mathrm{cm}^{-1}\right): 3465,3071,1606,1520,1344 ;{ }^{1} \mathrm{HNMR}\left(\mathrm{DMSO}_{-} \mathrm{d}_{6}, 300 \mathrm{MHz}\right): 8.50(2 \mathrm{H}, \mathrm{d}$, $\mathrm{J}=9.0 \mathrm{~Hz} \mathrm{Ar}), 8.60(2 \mathrm{H}, \mathrm{d}, \mathrm{J}=8.4 \mathrm{~Hz} \mathrm{Ar}), 8.51(2 \mathrm{H}, \mathrm{d}, \mathrm{J}=8.1 \mathrm{~Hz} \mathrm{Ar}), 7.79(2 \mathrm{H}, \mathrm{d}, \mathrm{J}=8.4 \mathrm{~Hz} \mathrm{Ar}), 3.15$ $\left(1 \mathrm{H}, \mathrm{dd}, \mathrm{J}=10.1 \mathrm{~Hz}, 9.6 \mathrm{~Hz}\right.$ C- $\left.\mathrm{H}_{2 \mathrm{a}}\right), 3.82\left(1 \mathrm{H}, \mathrm{dd}, \mathrm{J}=10.1 \mathrm{~Hz}, 9.6 \mathrm{~Hz} \mathrm{C}-\mathrm{H}_{2 \mathrm{~b}}\right), 5.15(1 \mathrm{H}, \mathrm{t}, \mathrm{J}=7.4 \mathrm{~Hz} \mathrm{C}-\mathrm{H})$, $7.99(1 \mathrm{H}, \mathrm{s}, \mathrm{N}-\mathrm{H}), 2.62\left(3 \mathrm{H}, \mathrm{s}, \mathrm{CH}_{3}\right):{ }^{13} \mathrm{C}$ NMR (DMSO-d $\left.{ }_{6}\right): 152.13,148.9,146.1,145.2,143.3$, 141.8, 137.1, 137.8, 132.9, 129.6, 128, 128.86, 128.24, 127.0, 126.9, 126.4, 125.1, 63.2, 43.9, 14.6; MS: $m / \mathrm{z} 366.79\left[\mathrm{M}^{+}\right]$.

11. 5-(5-(4-bromophenyl)-4,5-dihydro-1H-pyrazol-3-yl)-4-methyl-1-(4-nitrophenyl)-1H-1,2,3triazole (Pale Yellow Color Amorphous Solid)

MP $210^{\circ} \mathrm{C}$; IR( $\left.\mathrm{KBr}, \mathrm{cm}^{-1}\right): 3326,3084,1597,1531,1348 .{ }^{1} \mathrm{HNMR}\left(\mathrm{DMSO}-\mathrm{d}_{6}, 300 \mathrm{MHz}\right): 8.52(2 \mathrm{H}, \mathrm{d}$, $\mathrm{J}=10.2 \mathrm{~Hz} \mathrm{Ar}), 8.01(2 \mathrm{H}, \mathrm{d}, \mathrm{J}=9.0 \mathrm{~Hz} \mathrm{Ar}), 7.57(2 \mathrm{H}, \mathrm{d}, \mathrm{J}=8.4 \mathrm{~Hz} \mathrm{Ar}), 7.38(2 \mathrm{H}, \mathrm{d}, \mathrm{J}=8.4 \mathrm{~Hz} \mathrm{Ar}), 3.01$ $\left(1 \mathrm{H}, \mathrm{dd}, \mathrm{J}=10.2 \mathrm{~Hz}, 10.2 \mathrm{~Hz} \mathrm{C}-\mathrm{H}_{2 \mathrm{a}}\right), 3.67\left(1 \mathrm{H}, \mathrm{dd}, \mathrm{J}=10.8 \mathrm{~Hz}, 10.8 \mathrm{~Hz} \mathrm{C}-\mathrm{H}_{2 \mathrm{~b}}\right), 4.85(1 \mathrm{H}, \mathrm{t}, \mathrm{J}=7.4 \mathrm{~Hz} \mathrm{C}-$ $\mathrm{H}), 7.80(1 \mathrm{H}, \mathrm{s}, \mathrm{N}-\mathrm{H}), 2.61\left(3 \mathrm{H}, \mathrm{s}, \mathrm{CH}_{3}\right):{ }^{13} \mathrm{C}$ NMR $\left(\mathrm{DMSO}_{-} \mathrm{d}_{6}\right): 148.0,143.69,142.75,140.95$, $139.91,132.55,131.77,129.36,126.38,125.56,120.63,62.40,42.33,10.56 ; \mathrm{MS}: \mathrm{m} / \mathrm{z} 426.1\left[\mathrm{M}^{+}\right]$ $428.1[\mathrm{M}+2]$.

\section{RESULTS AND DISCUSSION}

The $\mathrm{p}$ - amido aniline derived chalcones have shown appreciable antimicrobial activity compared to the standard drugs. ${ }^{22,23}$ Literature review reveals that only very few 1, 2, 3- triazole linked pyrazolines have been synthesized. As per the procedure given in the literature ${ }^{19} 4$ - nitro aniline was converted into its corresponding 4- methyl-5-acetyl-1, 2, 3- triazole. In the present work pyrazoline was synthesized as given in the literature. ${ }^{24,25}$ The conversion of 4- nitro aniline into azide $\mathbf{1}$ was confirmed by $\mathrm{N}=\mathrm{N}$ stretching absorption band at $2127 \mathrm{~cm}^{-1}$ in its IR spectrum. The formation of 4- methyl- 1- 4- nitrophenyl$1 H$ - 1, 2, 3- triazole- 5-yl ethanone 2 was ensured from a three proton singlet that appeared at $\delta$ value 2.60 for the methyl group. The chalcones 3.1- 3.11 were synthesized from 5- acetyl- triazole. Two doublets at $\delta 7.92$ and 8.0 for $\mathrm{H} \alpha$ and $\mathrm{H} \beta$ protons revealed the formation of chalcone. A series of target compounds pyrazolines 4.1- 4.11 were synthesized by following the literature. ${ }^{24,25}$ The stereochemically non- equivalent proton signals at $\delta 3.01 \& 3.04$ and a triplet at $\delta 4.86$ confirm the formation of pyrazoline. The compounds 3-(4-methoxyphenyl)-1-(4-methyl-1-(4-nitrophenyl)-1H-1, 2, 3-triazol-5-yl) prop-2-en-1one, 3-(2-chlorophenyl)-1-(4-methyl-1-(4-nitrophenyl)-1H-1, 2, 3-triazol-5-yl) prop-2-en-1-one and 3-(4- 


\section{RASĀYAN J. Chem. \\ Vol. 13 | No. 1 |676-683| January - March | 2020}

nitrophenyl)-1-(4-methyl-1-(4-nitrophenyl)-1H-1, 2, 3-triazol-5-yl) prop-2-en-1-one have shown zone of inhibition against Klebsiella, E. faecalis , Pseudomonas fluorescence 14,16,16/20mm and the compounds 5-(5-(4-methoxyphenyl)-4, 5-dihydro-1H-pyrazol-3-yl)-4-methyl-1-(4-nitrophenyl)-1H-1, 2, 3-triazole, 4methyl-1-(4-nitrophenyl)-5-(5-(2-nitrophenyl)-4，5-dihydro-1H-pyrazol-3-yl)-1H-1，2，3-triazole have shown moderate inhibition against Klebsiella, E. faecalis, Pseudomonas fluorescence and S.aures $18,18,17,16 / 20 \mathrm{~mm}$. Appreciable antimicrobial activity was shown by the Methoxy, Chloro and Nitro substituted chalcones and pyrazolines against the standard drugs infer that polarity variation among the functional groups in the derived compounds also plays some vital role in addition to that of extending of conjugation.

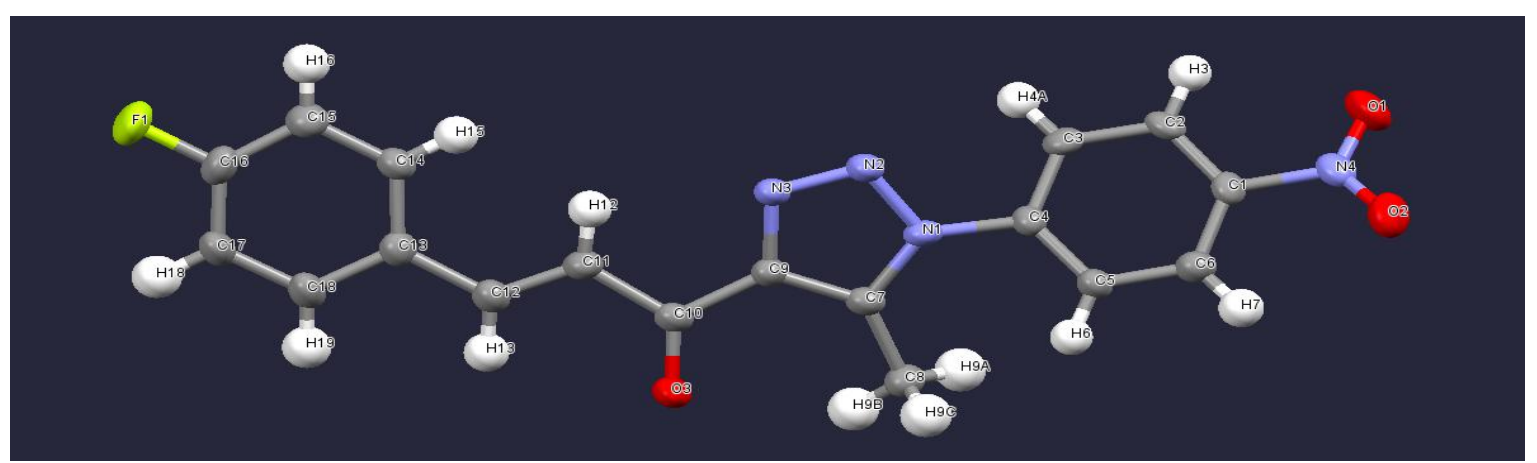

Fig.-1: XRD Molecular Structure of (E)-3-(4-fluorophenyl)-1-(4-methyl-1-(4-nitrophenyl)-1H-11, 2, 3-triazol-5-yl) prop-2-en-1-one

A single crystal of compound 3.10 was obtained by slow evaporation method at room temperature using an alcohol-chloroform mixture. The crystal structure was solved and refined using CrysAlisPro 1.171.39.46 (Rigaku Oxford Diffraction, 2018). Mo K $\alpha$ radiation $(\lambda=0.71073 \AA$ ) and a graphite monochromator were used for data collection. The chemical formula and labeling scheme is shown in Fig.-1. Crystal data for compound 3.10: $\mathrm{C}_{18} \mathrm{H}_{13} \mathrm{~N}_{4} \mathrm{O}_{3} \mathrm{~F}$; Mr. 352.32; system, triclinic; space group, 'P -1'; unit cell dimensions, a, 7.4413(8) Á; b, 7.5604(6) $\AA$; c, 15.4915(10) $\AA$; $\alpha, 87.980(6) ; \beta, 78.021(7)^{\circ} ; \gamma$, 67.884(9) ${ }^{\circ}$ V V , 788.95(11) A $\mathrm{A}^{3} ; \mathrm{Z}, 2 ; \mu, 0.112 \mathrm{~mm}^{-1}$; Density, $1.483 \mathrm{~g} \mathrm{~cm}^{-3} ; \theta$ range for data collection 2.714 - 29.801 ${ }^{\circ}$; T, $298 \mathrm{~K}$; independent reflections, 7413; measured reflections, 4391; used reflections, $1921 ; \mathrm{R}_{\mathrm{int}}, 0.0766 ; \mathrm{R}$ (all), 0.1318; Goodness of fit, 1.126 .

\section{CONCLUSION}

4-Nitro aniline derived 1, 2, 3 triazole linked pyrazolines were synthesized and evaluated for their antimicrobial activity. All the chalcones and pyrazolines have shown only moderate antimicrobial activity compared to the standard drugs streptozomycin and flucanazole. It was assumed that the extend of conjugation and electronegativity were the reasons for the better activity of the compounds. To conclude the above-said reason, the present work was designed with the precursor 4-nitro aniline.In our previous work, antimicrobial activity of 4-amino benzamide derived chalcones and pyrazolines were presented ${ }^{23}$. Comparison of antimicrobial activity of chalcones and pyrazolines of 4-nitro aniline derived compounds revealed that greater extend of conjugation and electronegativity is not reflected on the microbial activity of the synthesized compounds. So that incorporation of structural modification in the 1,2,3-triazolechalcone hybrid by incorporating the pyrazoline ring may not be a suitable chemical environment to achieve better anti-microbial compound was concluded.

\section{ACKNOWLEDGMENT}

The authors thank the SASTRA University and IIT Madras for providing spectral data and management of Vivekanandha Educational Institutions for providing laboratory facilities.

\section{REFERENCES}

1. Z. H. Lu, X. J. Gu, K. Z. Shi, X. Li, D. D. Chen, L. Chen, Arabian Journal of Chemistry, 10(5), 624 (2017),DOI: $10.1016 / j$.arabjc.2014.11.002

2. O. P. Pravin, B. B. Sanjay, Arabian Journal of Chemistry, 9, 588(2016), DOI: 
RASĀYAN J. Chem.

Vol. 13 | No. 1 |676-683| January - March | 2020

10.1016/j.arabjc.2013.08.0271878-5352

3. G. Vincent, F. Christophe, R. Millet, R. Mansouri, P. Chavatte, V. Levacher, J. F. Brière, European Journal of Medicinal Chemistry, 58, 396 (2012), DOI: 10.1016/j.ejmech.2012.10.031

4. R. Fioravanti, A. Bolasco, F. Manna, F. Rossi, F. Orallo, F. Ortuso, S. Alcaro, R. Cirilli, European Journal of Medicinal Chemistry, 45(12),6135 (2010), DOI:10.1016/j.ejmech.2010.10.005

5. H. Khalilullah, S. Khan, M. J. Ahsan, B. Ahmed, Bioorganic \&. Medicinal Chemistry Letters, 21 (24), 7251,( 2011), DOI: 10.1016/j.bmcl.2011.10.056

6. M. R. Bhosle, A. R. Deshmukh, S. Pal, A. K. Srivastava, R. A. Mane, Bioorganic \&. Medicinal Chemistry Letters, 25,2442 (2015), DOI:10.1016/j.bmcl.2015.03.068

7. S. Viveka, Dinesha, P. Shama, G. K. Nagaraja, S. Ballav, S. Kerkar, European Journal of Medicinal Chemistry, 101, 442(2015), DOI:10.1016/j.ejmech.2015.07.002

8. A. Adhikari, B. Kalluraya, K. V. Sujith, K. Gouthamchandra, R. Jairam, R. Mahmood, R. Sankolli, European Journal of Medicinal Chemistry, 55, 467(2012), DOI:10.1016/j.ejmech.2012.07.002

9. R. Aggarwal, S. Kumar, P. Kaushik, D. Kaushik, G. K. Gupta, European Journal of Medicinal Chemistry, 62, 508(2013), DOI:10.1016/j.ejmech.2012.11.046

10. B. N. Acharya, D. Saraswat, M. Tiwari, A. K. Shrivastava, R. Ghorpade, S. Bapna, M. P. Kaushik, European Journal of Medicinal Chemistry, 45(2), 430 (2010), DOI:10.1016/j.ejmech.2009.10.023

11. A. Adhikari, B. Kalluraya, K. V. Sujith, K. Gouthamchandra, R. Jairam, R. Mahmood, R. Sankolli, European Journal of Medicinal Chemistry, 55, 467 (2012), DOI:10.1016/j.ejmech.2012.07.002

12. E. A. Begum, Y. C. Samiye, U. Gulberg, Y. Kemal, E. Rahmiye, Bioorganic \&. Medicinal Chemistry Letters, 24, 3278(2014), DOI:10.1016/j.bmcl.2014.06.015

13. R. M. Kumbhare, T. L. Dadmal, M. JanakiRamaiah, K. S. V. Kishore, L. Pushpavalli, S. Tiwari., K. Appalanaidu, Y. KhageswarRao, M. Bhadra, Bioorganic \&. Medicinal Chemistry Letters, 25 (3), 654(2015), DOI:10.1016/j.bmcl.2014.11.083

14. C. K. Sharad, B. P. Vishal, K. R. Dipak, European Journal of Medicinal Chemistry, 84, 51(2014), DOI:10.1016/j.ejmech.2014.07.008

15. J. A. Mohamed, G. S. Jeyabalan, S. Savita, J. Naresh, K. Lalit, K. S. Lalit, Y. Hemant, S. Lokesh, G. K. Rajput, S. D. Narendra, P. Ravindra, B. J. Chandra, Bioorganic \&. Medicinal Chemistry Letters, 21, 5259(2011), DOI:10.1016/j.bmcl.2011.07.035

16. F. A. Bakr, A. Ehab, A. M. Hanan, E. A. A. Ghada, European Journal of Medicinal Chemistry, 52, 263(2012), DOI:10.1016/j.ejmech.2012.03.023

17. Y. W. Mohmmad, R. B. Abdul, A. Amir, H. L. Dae, C. Inho, A. Fareeda, European Journal of Medicinal Chemistry, 54, 845(2012), DOI: 10.1016/j.ejmech.2012.03.049

18. C. P. Kaushik, R. Luxmi, D. Singh, A. Kumar, Monatshefte für Chemie 2 21, 137(2017), DOI: 10.1007/s00706-019-2361-9

19. V. R. Kamalraj, S. Senthil, P. Kannan, Journal of Molecular Structure., 892, 210(2008), DOI: $10.1016 /$ j.molstruc.2008.05.028

20. N. T. Pokhodylo, R. D. Savka, V. S. Matiichuk, N. D. Obushak, Russian Journal of General Chemistry, 79, 309(2009), DOI:10.1134/S1070363209020248

21. H. S. Dong, H. C. Wang, Z. L. Gao, R. S. Li, F. H. Cui, Journal of Heterocyclclic Chemistry, 47, 389(2010), DOI:10.1002/jhet.336

22. N. Hari, S. Aravind,G. Kurian, R. Rajmohan, R. Ravikumar, Der Pharmazia Lettre, 8, 275(2016)

23. S. Sathiya, S. Nandhabala, N. Hari, R. Paranthaman, A. Sankar, R. Ravikumar, Rasayan Journal of Chemistry, 12 (4), 2260(2019), DOI:10.31788/RJC.2019.1245383

24. M. Malathi, D. Prabhakara Chary, Rasayan Journal of Chemistry, 12(3), 1347(2019), DOI: 10.31788/RJC.2019.1235290

25. M. Idrees, S. Kola, N. J. Siddiqui, Rasayan Journal of Chemistry, 12(4), 1725 (2019), DOI: 10.31788/RJC.2019.1245467

[RJC-5568/2019] 